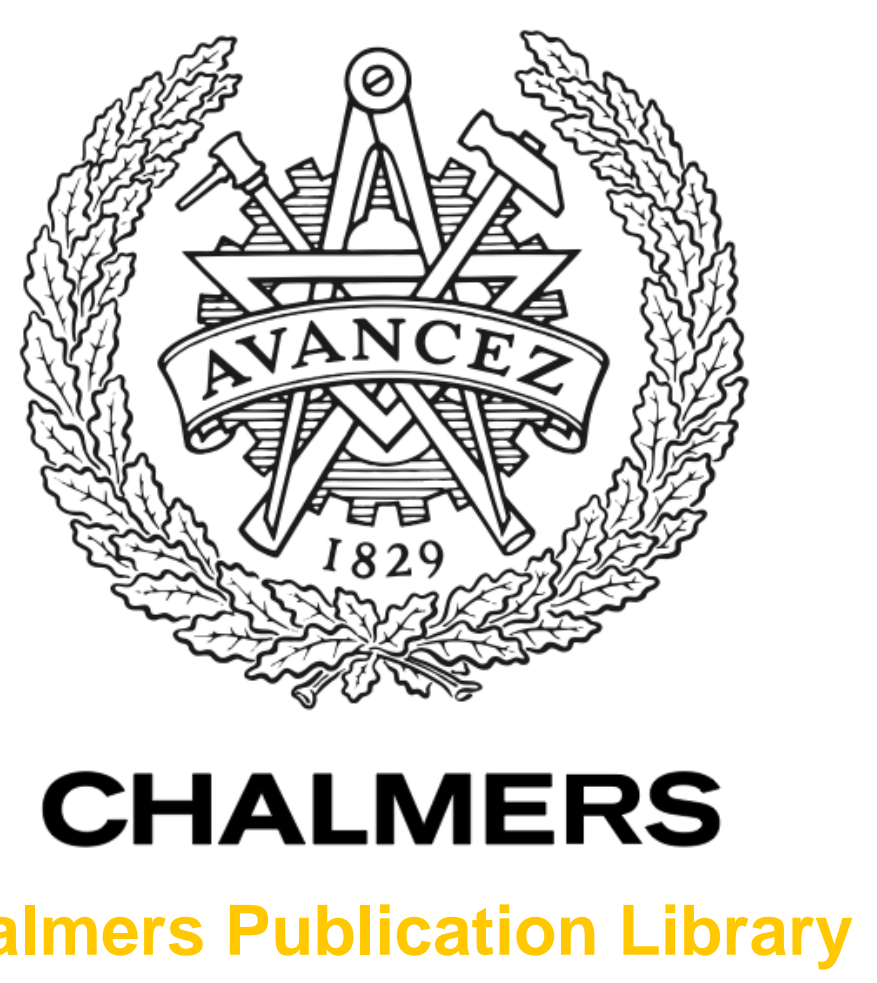

Chalmers Publication Library

\title{
Green and lean control of cyclic pallet systems
}

This document has been downloaded from Chalmers Publication Library (CPL). It is the author's version of a work that was accepted for publication in:

2011 7th IEEE International Conference on Automation Science and Engineering, CASE 2011; Trieste; 24 August 2011 through 27 August 2011 (ISSN: 21618070)

Citation for the published paper:

Mashaei, M. ; Lennartson, B. ; Abbestam, G. (2011) "Green and lean control of cyclic pallet systems". 2011 7th IEEE International Conference on Automation Science and Engineering, CASE 2011; Trieste; 24 August 2011 through 27 August 2011 pp. 642-647.

http://dx.doi.org/10.1109/CASE.2011.6042444

Downloaded from: http://publications.lib.chalmers.se/publication/150971

Notice: Changes introduced as a result of publishing processes such as copy-editing and formatting may not be reflected in this document. For a definitive version of this work, please refer to the published source. Please note that access to the published version might require a subscription.

Chalmers Publication Library (CPL) offers the possibility of retrieving research publications produced at Chalmers University of Technology. It covers all types of publications: articles, dissertations, licentiate theses, masters theses, conference papers, reports etc. Since 2006 it is the official tool for Chalmers official publication statistics. To ensure that Chalmers research results are disseminated as widely as possible, an Open Access Policy has been adopted.

The CPL service is administrated and maintained by Chalmers Library. 


\title{
Green and Lean Control of Cyclic Pallet Systems
}

\author{
Maziar Mashaei $^{1,2}$, Bengt Lennartson ${ }^{1}$, Göran Abbestam ${ }^{2}$ \\ ${ }^{1}$ Department of Signals and Systems, Chalmers University of Technology, Sweden \\ ${ }^{2}$ FlexLink AB SE-415 50 Göteborg Sweden
}

\begin{abstract}
Reduction of energy consumed by a manufacturing system to turn raw parts to finished products is a big step towards the green and lean production. In this study the energy efficiency of a one-loop pallet system, a main tool to handle and locate various part types in a cyclic production line, is investigated. The main goal is to obtain the minimal energy consumption in the pallet system drive unit through an optimally controlled and coordinated motion of pallets. To achieve the mentioned goal, first the mean value of the pallet system energy consumption is mathematically modeled. Later, this energy model is utilized as an objective function within an optimization model including constraints on system crucial properties such as cyclic and dynamic behavior, queueing policy, and buffer size. The solution of the optimization problem gives the optimal values of the system control variables, namely, number of pallets and conveyor velocity. To demonstrate the application of this optimization model in practice, three case studies are introduced. The results of these studies show that a significant amount of the energy consumption may be saved by applying the suggested green control.
\end{abstract}

Key words: Handling and locating pallet system, Energy model, Optimization.

\section{INTRODUCTION}

The green manufacturing paradigm includes designs and strategies which minimize the impact of production systems on environment [1]. On the other hand, lean production is a method of doing business based on gaining profit through eliminating the waste in production sectors [2]. Both these concepts promote the energy efficiency as an important key in the design of manufacturing plants, although they may introduce different strategies towards this design goal.

The consumed energy in a manufacturing system can be attributed to equipment technology and type of processes as well as the coordination of operations on the system level. This classification is important because equipment and process energy efficiency demand a proper selection of equipments and related processes in a plant design. System energy efficiency, on the other hand, corresponds to an optimal policy for the coordination of operations in a control design.

This paper focuses on the problem of designing an optimal control for one-loop pallet systems. The goal is to provide the desired cycle time for the production of specific parts (just in time strategy), as well as to maintain minimal energy consumption for the system drive unit (green production).

\section{A. Handling and locating pallet systems}

A pallet system governs processing operations by directing and locating the parts to be processed. Beside the pallets, which are carrier of the parts, two other major components in a pallet system are conveyor lines and function modules. The conveyor line transports and buffers the pallets, and the function module locates the pallets for loading, unloading, and processing actions. In a one-loop pallet system, the parts are loaded and fixated on the pallets in the loading module. Subsequently, conveyor lines conduct the pallets to locating modules where they are positioned, and their parts are operated by processing machines or robots. After finishing all machining stages, the pallets enter to the unloading module, and the completed parts (products) are unloaded in this module. Finally, the empty pallets are redirected to the loading module, and one closed loop motion of pallets takes form.

One-loop pallet systems are often used in cyclic production lines with multiple part types. A production line is cyclic if it periodically produces a certain set of parts in a specific duration denoted cycle time. One-loop pallet systems for cyclic production lines are from now called cyclic pallet systems. In a cyclic pallet system, although all parts pass the same order of modules from the loading to unloading section, their processing times and process sequences can be different with respect to their types. Figure 1 shows a cyclic pallet system with the set of parts $\{A, B 1, B 2\}$ and the function modules $\left\{M^{1}, \ldots, M^{6}\right\}$. The sequence of operations for these parts are defined as follows

- $A: M^{1} \rightarrow M^{2} \rightarrow M^{6}$

- $B 1: M^{1} \rightarrow M^{3} \rightarrow M^{5} \rightarrow M^{6}$

- $B 2: M^{1} \rightarrow M^{4} \rightarrow M^{5} \rightarrow M^{6}$

This means that for instance, part $B 1$ is loaded in $M^{1}$, located in the modules $\left\{M^{3}, M^{5}\right\}$ for processing operations, and unloaded in $M^{6}$.

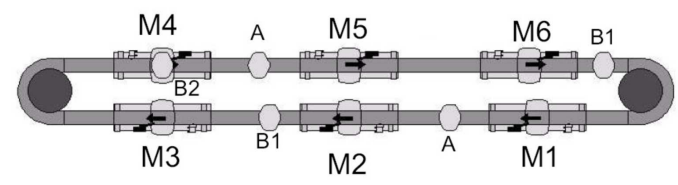

Fig. 1. One closed loop pallet system

\section{B. Challenges in control of cyclic pallet systems}

A cyclic pallet system with one part type can be modeled by a Petri Net formalism [3]. In this modeling framework, the tokens represents the pallets, and the places and the transitions correspond to the buffers and the locating modules, 
respectively. For the obtained model, Ramamoorthy et al [4] developed the main equation relating the number of tokens (pallets) $N_{p}$, the cycle time $C$, and the flow time of a pallet moving one loop in the system, $F T$, as

$$
C=\frac{F T}{N_{p}}
$$

When a set of various parts is periodically handled in the pallet system, according to [5] and [6], the above equation is still held. However, in the latter case $F T$ is considered as overall flow times for the multiple parts in the set, and the cycle time denotes the average production time of the set of parts.

Fulfillment of a desired cycle time is the main purpose to control a cyclic pallet system. Based on the lean philosophy, the control set points should be parameterized in an optimal way to reduce the flow time as well as the number of pallets. Since $F T$ is an increasing function of $N_{p}$, this optimal control is obtained through the minimization of the number of pallets with respect to a specified cycle time (the minimal number of pallets). Many studies have investigated such a control with different methods and different characteristics of the production system.

For nondeterministic closed loop production lines with unreliable machines, analytical methods based on probability analysis are the main tools for the system performance evaluation [7]. Frein et al [8] and Gershwin et al [9] used decomposition techniques to evaluate the line production rate by considering a limited number of pallets. Han et al [10] applied a Taylor series expansion, and Biller et al [11] assumed a Bernoulli reliability model for machines to obtain a relation between buffer sizes and the minimal number of pallets.

Regarding deterministic production lines, optimization models based on marked graph Petri Nets is the dominant approach [4]. Magott et al [12] and Yamada et al [13] formulated linear programming models based on Ramamoorthy's result to obtain the minimum cycle time in the marked graph Petri Nets. Korbaa et al [14], Chauvet et al [15], and Hsu et al [16] furthermore developed the heuristic and formal optimization methods to minimize the cycle time as well as the work in process (number of pallets).

One interesting question which may arise is if the minimal number of pallets demands the minimum driving energy as well or not. Moreover, if it does not, what number of pallets under which control policy fulfills this green property for the pallet system. To the best of the authors' knowledge, no studies have been addressed to concern these questions. This paper provides a method to investigate and answer these questions for deterministic cyclic pallet systems.

\section{Assumptions, Notations, AND Definitions}

All mathematical models in this paper are developed based on the following assumptions, definitions, and notations.

\section{Assumptions:}

- Pallet system has only one loading, unloading, and drive unit.

- One pallet can handle only one product at a time, and the product remains clamped to the pallet during its entire journey in a pallet system.

- Processing times and transportation times are deterministic, and there is no failure in machines and function modules.

- The conveyor line transports pallets in a horizontal direction (there is no slope for conveyor lines).

- Empty pallets are reloaded with new parts as soon as possible. Besides, the pallet waiting times in the loading queue are negligible.

- Universal (the same type of) pallets are used for different types of products.

- An assigned operation cannot be removed from a module until it is completed.

- A single resource can only be used for one operation at a time.

- All parts are removed when they reach the unloading section (no re-entrance).

- All function modules follow the FIFO (first input first output) policy.

- The pallet is disconnected from the conveyor chain when the pallet is located.

- There is a fixed and known schedule for the entrance of different part types to the pallet system.

- A wheel type bend is used to change the orientation of a conveyor chain. This kind of bend nearly introduces zero friction between the chain and the bend slide.

\section{Definitions:}

Definition 1. (Module segment): A cyclic pallet system is partitioned into module segments. Each segment includes one locating module and its related conveyor line for buffering the pallets behind the module.

Definition 2. (Starving and waiting time): Starving time is the duration time in which a module is in an idle state before receiving a pallet. Waiting time is the time one pallet has to wait in a queue of a segment.

Definition 3. (Minimal part set): Let $n_{u}$ denote the number of parts that correspond to product type $u$ in the overall production target. Suppose there are $h$ different product types. If $q$ is the greatest common divisor of the integers $\left\{n_{1}, \ldots, n_{h}\right\}$, then the vector $\bar{n}=\left(\frac{n_{1}}{q}, \ldots, \frac{n_{h}}{q}\right)$ represents the smallest set having the same proportions of the different product types as the production target. This set is usually referred to as the Minimum Part Set (MPS) (this definition is exactly brought from [17]).

Definition 4. (Drive unit of a pallet system): A drive unit transports pallets in the system and consists of an electrical motor, a conveyor chain, and related accessories (motor speed convertor, electrical driver, etc).

\section{Notations:}

- $\ell \in M=\{1,2, \ldots, m\}$ is the index over all modules. 
Moreover, $\ell=1$ and $\ell=m$ denote loading and unloading modules, respectively.

- $N^{\ell}(t)$ is the number of pallets at time $t$ in the segment which includes the module $\ell$. In addition, $N_{p}=$ $\sum_{\ell=1}^{m} N^{\ell}(t)$ is the total number of pallets in the system.

- $L^{\ell}$ denotes the length of the segment related to the module $\ell$, and $b^{\ell}$ is the size of the buffer in this segment (including the locating space). Moreover, $L=\sum_{\ell=1}^{m} L^{\ell}$ is the overall length of the pallet system.

- $i \in I=\{1, \ldots, n\}$ is the index over parts $\Pi=$ $\left\langle P_{1}, P_{2}, \ldots P_{n}\right\rangle$ in a minimal part set.

- $\tau_{i}^{\ell}$ is the processing time in module $\ell$ for $P_{i}$.

- $T_{i}$ is the total handling time for $P_{i}$ from the loading module to the unloading one. $T_{i}$ includes the processing times, transportation times, and waiting times for the part.

- $T^{m 1}$ is empty pallet transportation time from the unloading to the loading section. Besides, $T_{i}+T^{m 1}$ defines the flow time for $P_{i}$.

- $v_{c}[\mathrm{~m} / \mathrm{s}]$ denotes the conveyor velocity and $T_{c}=1 / v_{c}$ is the transportation time for one pallet passing 1 meter.

- $k \in K=\left\{1,2, \ldots k^{s}, k^{s}+1\right\}$ is the index over all cycles in transition states and the first $\left(k^{s}\right)$ and the second $\left(k^{s}+\right.$ 1) cycle in which the system enters to the steady state phase.

- $S_{i}^{\ell}[k]$ is the time when $P_{i}$ is released by module $\ell$ in cycle $k$.

- $C_{i}^{\ell}=S_{i+1}^{\ell}[k]-S_{i}^{\ell}[k]$ for $i \in I \backslash\{n\}$ and $k \geq k^{s}$, and $C_{n}^{\ell}=$ $S_{1}^{\ell}[k+1]-S_{n}^{\ell}[k]$ for $k \geq k^{s}$ are defined as steady state interval times.

- $m_{c}$ is the mass of one meter chain $[\mathrm{kg} / \mathrm{m}]$ and $m_{p}$ is the average mass for a part (including its pallet) in a pallet system.

- $\mu_{s c}$ is the friction coefficient between the conveyor slide and conveyor chain, and $\mu_{c p}$ is the friction coefficient between the conveyor chain and a pallet.

- $\mathbb{N}, \mathbb{Z}$, and $\mathbb{R}$ denote the domain of variables for Natural $+\{0\}$, Integer, and Real numbers, respectively.

\section{ENERGY MODEL FOR THE MEAN VALUE ENERGY CONSUMPTION}

In a cyclic pallet system, pallets are transported by a conveyor chain, which is connected to an electrical motor. Assuming that the shaft of the motor is attached to the conveyor chain at the exit of the unloading segment, the total drive system energy consumption $E(t)$ during $[0, t]$ is calculated by

$$
E(t)=\frac{1}{\eta} \int_{0}^{t} F^{m}(s) v_{c} d s
$$

Here, $F^{m}(t)$ is the chain tension force at the exit of the unloading module, and $\eta \simeq 0.8$ is the drive unit efficiency.

$N^{\ell}(t)$ is the sum of the number of pallets staying at the buffer, $N_{s}^{\ell}(t)$, and those being transported in the segment, $N_{\text {mov }}^{\ell}(t)$. Therefore, the friction force initiated from the weight of the conveyor chain and the pallets (with products) in segment $\ell$ at time $t$ is

$f^{\ell}(t)=\left(\left(N^{\ell}(t)-N_{f}^{\ell}(t)\right) m_{p}+L^{\ell} m_{c}\right) g \mu_{s c}+\left(N_{s}^{\ell}(t)-N_{f}^{\ell}(t)\right) m_{p} g \mu_{c p}$

where

$$
N_{f}^{\ell}(t)= \begin{cases}1 & \text { a pallet is located in the module } \ell \text { at } t \\ 0 & \text { a pallet is not located in the module } \ell \text { at } t\end{cases}
$$

Consequently, the tension force in the chain at the exit of the unloading module $(\ell=m)$ is obtained by $F^{m}(t)=\sum_{\ell=1}^{m} f^{\ell}(t)$ which leads to

$$
\begin{aligned}
F^{m}(t) & =\left(N_{p} m_{p}+L m_{c}\right) g \mu_{s c}+m_{p} g \mu_{c p} \sum_{\ell=1}^{m} N_{s}^{\ell}(t) \\
& -m_{p} g\left(\mu_{s c}+\mu_{c p}\right) \sum_{\ell=1}^{m} N_{f}^{\ell}(t)
\end{aligned}
$$

The term $\sum_{\ell=1}^{m} N_{s}^{\ell}(t)$ is a complex function, and its determination requires a precise dynamic model of the pallet system at any given $t$. Considering the lack of this model, a randomness pattern is assumed in $\sum_{\ell=1}^{m} N_{s}^{\ell}(t)$, and the following lemma is devised to find its mean value.

Lemma 1: The mean value of the number of mobile pallets in the pallet system is $n L T_{c} / C$.

Proof: The probability that one pallet is in a mobile condition can be obtained by $p=L T_{c} /\left(T^{m 1}+\frac{1}{n} \sum_{i=1}^{n} T_{i}\right)$. Here, $L T_{c}$ is the overall time one pallet is running one loop in the pallet system (without including process and waiting times), and $T^{m 1}+\frac{1}{n} \sum_{i=1}^{n} T_{i}$ is the average flow time of one pallet over various parts in an MPS. Because each pallet is either in a mobile or stationary state, a binomial distribution with respect to motion state of the pallets may be formed as $B_{n}\left(N_{p}, p\right)$. Therefore, the mean value for the number of mobile pallets, $N_{m o v}$, is estimated by

$$
N_{\text {mov }}=N_{p} p=N_{p} \frac{n L T_{c}}{n T^{m 1}+\sum_{i=1}^{n} T_{i}}
$$

Besides, (1) for a cyclic pallet system can be written as

$$
N_{p} C=n T^{m 1}+\sum_{i=1}^{n} T_{i}
$$

where the right side is the overall flow times for the multiple parts in the set. Substituting $N_{p} C$ in (5) gives $N_{m o v}=\frac{n L T_{c}}{C} . \square$

The mean value of $\sum_{\ell=1}^{m} N_{s}^{\ell}(t)$ is now used to determine the mean value energy consumption for one cycle of production.

Theorem 1: The mean value of the energy consumption in a drive unit of the cyclic pallet system for one production cycle has the form

$$
E\left(N_{p}, T_{c}\right)=\frac{a_{1} N_{p}+a_{2}}{T_{c}}+a_{3}
$$

where $a_{1}, a_{2}$, and $a_{3}$ are constant coefficients.

Proof: The mean value of $\sum_{\ell=1}^{m} N_{s}^{\ell}(t)$ can be calculated by $N_{p}-N_{m o v}$. Accordingly, based on Lemma 1 and the relation (4), the mean value of the energy consumption for one production cycle $(t=C)$ becomes

$$
E\left(N_{p}, T_{c}\right)=\frac{1}{\eta}\left(\frac{C\left(c_{1} N_{p}+c_{2}\right)}{T_{c}}-c_{3}-\frac{c_{1}}{T_{c}} \sum_{\ell=1}^{m} \int_{0}^{C} N_{f}^{\ell}(t) d t\right)
$$


where $c_{1}=m_{p} g\left(\mu_{c s}+\mu_{c p}\right), \quad c_{2}=L m_{c} g \mu_{c s}, \quad$ and $c_{3}=$ $n L m_{p} g \mu_{c p}$. The cyclic property of the pallet system in the steady state imposes the condition $\sum_{i=1}^{n} C_{i}^{\ell}=C$. Thus, the integral term in (8) can be calculated as follows

$$
\begin{aligned}
\int_{0}^{C} N_{f}^{\ell}(t) d t & =\int_{0}^{C_{1}} N_{f}^{\ell}(t) d t+\int_{C_{1}}^{C_{1}+C_{2}} N_{f}^{\ell}(t) d t+\cdots \\
& =\tau_{1}^{\ell}+\tau_{2}^{\ell}+\cdots+\tau_{n}^{\ell}=\sum_{i=1}^{n} \tau_{i}^{\ell}
\end{aligned}
$$

Substitute this term in (8) and define $\tau=\sum_{\ell=1}^{m} \sum_{i=1}^{n} \tau_{i}^{\ell}$, the equation (8) is modified to

$$
E\left(N_{p}, T_{c}\right)=\frac{1}{\eta}\left(c_{1} \frac{C N_{p}}{T_{c}}+\frac{C c_{2}-c_{1} \tau}{T_{c}}-c_{3}\right)
$$

which implies (7).

Corollary 1: Provided that $N_{p}$ is a constant value, (7) is a strictly decreasing function of $T_{c}$.

Proof: It is only needed to prove that the relation $a_{1} N_{p}+a_{2}>0$ is always met. According to (6) and (9),

$a_{1} N_{p}+a_{2}=\frac{1}{\eta}\left(c_{1}\left(-\tau+n T^{m 1}+\sum_{i=1}^{n} T_{i}\right)+C c_{2}\right)>0$

In the energy models $N_{p}$ and $T_{c}$ can not take any arbitrary value. To fulfill the cycle time $C$, these parameters should be selected from the domains specified by a set of pallet system design constraints. In the next section, these constraints are introduced.

\section{MAThematiCAL MOdELS OF THE CYCliC PALLET SYSTEM}

A pallet system is a closed queuing network in which pallets are entities in the segment queues. In [18], the authors developed the mathematical models, which characterize the dynamic behavior of a cyclic pallet system. Employing these models as the constraints in an optimization problem, the problem solution gives the level of queues at any given time in the cyclic period. The goal is to optimally control these levels to achieve the minimal energy consumption of the drive unit for the desired cycle time. In this section, first the design constraints are addressed, then this optimal control is characterized through an optimization model based on these constraints. The optimization model solution realizes the green and lean control of the cyclic pallet system.

\section{A. Design constraint models}

A coordination of operations in a cyclic pallet system is subjected to various constraints, due to scheduling, dynamic, cyclic, pallet, and buffer properties. In the below, these constraints are addressed based on [18].

Scheduling constraints : To consider the impact of parts scheduling on the dynamics of operations, the permutation matrix $\Delta$ is defined as an operator to map the initial sequence of part operations in the pallet system modules to the desired sequence of the operations. Denoting $[\Delta]_{i, j}=\delta_{i, j}$, this mapping can be expressed as

$$
\begin{aligned}
& i, j \in I, \quad \ell \in M \\
& \sum_{i=1}^{n} \delta_{i, j}=1, \quad \sum_{j=1}^{n} \delta_{i, j}=1, \quad \sum_{j=1}^{n} \delta_{i, j} \tau_{j}^{\ell}=T_{i}^{\ell}
\end{aligned}
$$

Dynamic constraints: These constraints introduce a relation among the part release times $S_{i}^{\ell}[k]$, the transportation times $L^{\ell} T_{c}$, and the operating times $T_{i}^{\ell}$ based on the consecutive order of types, modules, and cycles $(i, \ell, k)$.

$$
\begin{aligned}
& i \in I, \quad k \in K, \quad \ell \in M \\
& S_{i}^{\ell}[k]-S_{i}^{\ell-1}[k] \geq T_{i}^{\ell}+L^{\ell} T_{c} \quad: \quad \ell \in M \backslash\{1\} \\
& S_{i}^{\ell}[k]-S_{i-1}^{\ell}[k] \geq T_{i}^{\ell}: \quad i \in I \backslash\{1\} \\
& S_{1}^{\ell}[k+1]-S_{n}^{\ell}[k] \geq T_{1}^{\ell} \quad: \quad k \in K \backslash\left\{k^{s}+1\right\} \\
& S_{1}^{1}[1] \geq T_{1}^{1}
\end{aligned}
$$

Cyclic constraints: In the pallet system steady state, there is a cyclic pattern for the pallet releasing actions in each locating module. Assuming that the cyclic pattern will be started after the production cycle $k_{s}-1$, the part release times can be related to the cycle time $C$ and the interval times in each module $\left(C_{i}^{\ell} \geq T_{i}^{\ell}\right)$ as follows.

$$
\begin{aligned}
& k \in\left\{k^{s}, k^{s}+1\right\}, \quad \ell \in M \\
& S_{i+1}^{\ell}[k]-S_{i}^{\ell}[k]=C_{i}^{\ell}: \quad i \in I \backslash\{n\} \\
& S_{1}^{\ell}\left[k^{s}+1\right]-S_{n}^{\ell}\left[k^{s}\right]=C_{n}^{\ell} \\
& \sum_{i=1}^{n} C_{i}^{\ell}=C
\end{aligned}
$$

Pallet constraints: The pallet constraints model the closed loop behavior of a pallet motion in the system. Regarding that the number of pallets is a known parameter or a variable, two types of pallet constraints can be formed.

Pallet constraints type 1 ( $N_{p}$ is a known parameter):

$$
\begin{aligned}
& i \in I ; k \in\left\{k^{s}, k^{s}+1\right\} \\
& \sum_{e=1}^{N_{p}} C_{g(i, e)}^{m}=S_{i}^{m}[k]-S_{i}^{1}[k]+T_{i}^{1}+L^{1} T_{c}
\end{aligned}
$$

where

$$
g(i, e)=\left\{\begin{array}{lll}
n: & & i-e=n k, k \in \mathbb{Z} \\
(i-e) \quad \bmod n: & i-e \neq n k, k \in \mathbb{Z}
\end{array}\right.
$$

Pallet constraints type $2\left(N_{p}\right.$ is a variable):

$$
\begin{aligned}
& i \in I, \quad e \in I, \quad k \in\left\{k^{s}, k^{s}+1\right\} \\
& C N_{1}+r_{i}=S_{i}^{m}[k]-S_{i}^{1}[k]+T_{i}^{1}+L_{1} T_{c} \\
& r_{i}=\sum_{e=1}^{n} d_{i, e} \\
& C_{g(i, e)}^{m} \geq d_{i, e} \\
& d_{i, e} \geq C_{g(i, e)}^{m}-\left(1-x_{i, e}\right) C \\
& C x_{i, e} \geq d_{i, e} \\
& x_{i, e} \geq x_{i, e+1}: \quad e \in I \backslash\{n\} \\
& N_{p}=q n+\sum_{e=1}^{n} x_{i, e}
\end{aligned}
$$


Buffer constraints: $S_{i}^{\ell}[k]-S_{i}^{\ell-1}[k]$ specifies the duration in which part $i$ stays in segment $\ell$. According to (13), the constraint $\sum_{e=1}^{b^{\ell}} C_{g(i, e)}^{\ell}=S_{i}^{\ell}[k]-S_{i}^{\ell-1}[k]$ always requires $b^{\ell}$ number of pallets in the segment $\ell$. To let $N^{\ell}(t) \leq b^{\ell}$, this constraint is modified to

$$
\begin{aligned}
& i \in I, \quad \ell \in M \backslash\{1\}, k \in\left\{k^{s}, k^{s}+1\right\} \\
& \sum_{e=1}^{b^{\ell}} C_{g(i, e)}^{\ell} \geq S_{i}^{\ell}[k]-S_{i}^{\ell-1}[k]
\end{aligned}
$$

Variable constraints: All variables shall be clarified based on their domains such as binary, natural, and real. For the variables applied in the set of design constraints $D=\{(10),(11),(12),(13),(15),(16)\}$, the set of variables $V=\left\{N_{p}, N_{1}, T_{i}^{\ell}, S_{i}^{\ell}[k], T_{c}, C_{i}^{\ell}, r_{i}, d_{i, e}, \delta_{i, j}, x_{i, e}\right\}$ with the following domains are defined.

$$
\begin{aligned}
& i \in I, \quad j \in I, \quad e \in I, \quad \ell \in M, k \in K \\
& N_{p}, N_{1} \in \mathbb{N} \\
& T_{i}^{\ell}, S_{i}^{\ell}[k], T_{c}, C_{i}^{\ell}, r_{i}, d_{i, e} \in \mathbb{R} \\
& x_{i, e} \in\{0,1\}
\end{aligned}
$$

\section{B. Optimization models}

Let's assume $N_{p}^{*}$ and $T_{c}^{*}$ are the optimal values giving the minimal energy consumption for a cyclic pallet system with a desired cycle time $C$. This means that $E\left(N_{p}^{*}, T_{c}^{*}\right) \leq E\left(N_{p}, T_{c}\right)$ for any $N_{p}$ and $T_{c}$ satisfying the system design constraints. The optimal value $N_{p}^{*}$ and $T_{c}^{*}$ can be found by solving the following nonlinear optimization problem.

OP1-Mixed Integer Nonlinear Problem (MINLP) for obtaining the minimal energy consumption of a pallet system drive unit with the desired cycle time $C$ and the permutation matrix $\Delta$ :

$$
\begin{aligned}
& E^{*}=E\left(N_{p}^{*}, T_{c}^{*}\right)=\min \frac{1}{\eta}\left(c_{1} \frac{C N_{p}}{T_{c}}+\frac{C c_{2}-c_{1} \tau}{T_{c}}-c_{3}\right) \\
& \text { subject to }(D \backslash\{(13)\}) \bigcup(\{(17)\})
\end{aligned}
$$

The nonlinearity of the objective function may impose a huge computation time for this problem. To tackle this complexity, we suggest a mechanism of mapping OP1 into a set of linear optimization problems which their solutions embrace $N_{p}^{*}$ and $T_{c}^{*}$. This mechanism is realized with the following steps

1- A domain of candidates for $T_{c}^{*}$ is specified as $T_{c}^{*} \in$ $D_{T}=\left[0, T_{c}^{U}\right]$, where $T_{c}^{U}$ is obtained through the solution of $O P 2$.

OP2-Mixed Integer Linear Problem (MILP) for obtaining the minimal conveyor velocity (the maximal $T_{c}$ ) with the desired cycle time $C$ and the permutation matrix $\Delta$ :

$$
\begin{aligned}
& T_{c}^{U}=\max T_{c} \\
& \text { subject to }(D \backslash\{(13)\}) \bigcup(\{(17)\})
\end{aligned}
$$

2- A domain of candidates for $N_{p}^{*}$ is specified as $N_{p}^{*} \in D_{N}=\left[N_{p}^{\min }(0), N_{p}^{\min }\left(T_{c}^{U}\right)\right]$, where the boundaries of this domain are obtained through devising $O P 3$.
OP3-MILP for obtaining the minimal number of pallets with the desired cycle time $C$, the permutation matrix $\Delta$, and the conveyor velocity $T_{c}$ :

$$
\begin{aligned}
& N_{p}^{\min }\left(T_{c}\right)=\min \quad N_{p} \\
& \text { subject to } \\
& (D \backslash\{(13)\}) \bigcup\left(\left\{(17) \backslash\left\{T_{c}\right\}\right\}\right)
\end{aligned}
$$

3- According to Corollary 1, provided that the number of pallets is fixed, the minimal energy consumption is achieved when $T_{c}$ reaches to its maximum value. The optimization model, which characterizes this value, $T_{c}^{\max }\left(N_{p}\right)$, is

OP4-Linear program model for obtaining the maximum $T_{c}$ with the desired cycle time $C$, the permutation matrix $\Delta$, and the number of pallets $N_{p}$ :

$$
T_{c}^{\max }\left(N_{p}\right)=\max \quad T_{c}
$$

subject to

$$
(D \backslash\{(15)\}) \bigcup\left(\left\{(17) \backslash\left\{N_{p}, N 1, r_{i}, d_{i, e}, x_{i, e}\right\}\right\}\right)
$$

Consequently, $N_{p}^{*}, T_{c}^{*}$, and $E\left(N_{p}^{*}, T_{c}^{*}\right)$ can be realized through the search

$$
E\left(N_{p}^{*}, T_{c}^{*}\right)=\min \left\{E\left(N_{p}, T_{c}^{\max }\left(N_{p}\right)\right): N_{p} \in D_{N}\right\}
$$

In the next section, this three-step approach, which realizes the green and lean control of cyclic pallet systems, is applied to three case studies.

\section{ILlustrative EXAMPLES}

To demonstrate the application of the green and lean control in practice, we select three industrial pallet system technologies X85, XK, and XT as the test beds. These technologies, whose specification data are shown in Table I, have been designed and developed by FlexLink company [19]. Based on each technology, we design a pallet system similar to Fig. 1 to transport four parts $A, B 1, B 2, C$ among 6 function modules. Table II provides the design data including the processing times, the segment buffer sizes and lengths, and the desired cycle time for each pallet system. Furthermore, the unique scheduling matrix

$$
\Delta^{*}=\left(\begin{array}{cccc}
0 & 0 & 1 & 0 \\
0 & 1 & 0 & 0 \\
0 & 0 & 0 & 1 \\
1 & 0 & 0 & 0
\end{array}\right)
$$

is considered for these systems. To calculate the optimal control values of $N_{p}$ and $T_{c}$, we employ the mentioned steps in the previous section and obtain Table III. For

TABLE I

SPECIFICATION DATA FOR THREE PALLET SYSTEMS TECHNOLOGIES

\begin{tabular}{cccccc}
\hline \hline & & & & & \\
Tech & $m_{P}[k g]$ & $m_{c}\left[\frac{k g}{m}\right]$ & $\mu_{c p}$ & $\mu_{c s}$ & $\eta$ \\
\hline X85 & 10 & 1.25 & 0.3 & 0.1 & 0.8 \\
XK & 20 & 2.4 & 0.3 & 0.1 & 0.8 \\
XT & 30 & 1.25 & 0.3 & 0.15 & 0.8
\end{tabular}

each pallet system in this table, the minimal number of pallets, by which the system gains the desired cycle time, is specified by the smallest $N_{p}$. Furthermore, the values of $N_{p}^{*}$, $T_{c}^{*}$, and the related minimal energy consumption, which are 
TABLE II

PALlET SYSTEM DESIGN DATA

\begin{tabular}{ccccccccc}
\hline \hline & & & & & & & & \\
Tech & $M^{\ell}$ & $\tau_{A}[s]$ & $\tau_{B 1}[s]$ & $\tau_{B 2}[s]$ & $\tau_{C}[s]$ & $L^{\ell}[m]$ & $b^{\ell}$ & $C[s]$ \\
\hline & $M^{1}$ & 5 & 5 & 5 & 5 & 2 & 6 & \\
& $M^{2}$ & 15 & 10 & 0 & 25 & 1.5 & 4 & \\
X85 & $M^{3}$ & 0 & 18 & 22 & 0 & 1 & 2 & 55 \\
& $M^{4}$ & 20 & 0 & 0 & 15 & 1.5 & 4 & \\
& $M^{5}$ & 0 & 12 & 25 & 0 & 1 & 2 & \\
& $M^{6}$ & 5 & 5 & 5 & 5 & 1 & 2 & \\
\hline \multirow{4}{*}{ XK } & $M^{1}$ & 10 & 10 & 10 & 10 & 2 & 5 & \\
& $M^{2}$ & 0 & 35 & 0 & 25 & 1.5 & 3 & \\
& $M^{3}$ & 50 & 0 & 30 & 0 & 1 & 2 & 100 \\
& $M^{4}$ & 20 & 0 & 30 & 20 & 1.5 & 3 & \\
& $M^{5}$ & 20 & 45 & 0 & 20 & 1.5 & 3 & \\
& $M^{6}$ & 10 & 10 & 10 & 10 & 1 & 2 & \\
\hline \multirow{6}{*}{ XT } & $M^{1}$ & 10 & 10 & 10 & 10 & 3 & 6 & \\
& $M^{2}$ & 40 & 50 & 0 & 30 & 2 & 3 & \\
& $M^{3}$ & 60 & 0 & 45 & 0 & 1.5 & 2 & 160 \\
& $M^{4}$ & 0 & 45 & 0 & 35 & 2 & 3 & \\
& $M^{5}$ & 55 & 35 & 35 & 0 & 2 & 3 & \\
& $M^{6}$ & 10 & 10 & 10 & 10 & 1.5 & 2 &
\end{tabular}

set through the green and lean control, are distinguished with the bold fonts in the table. For example, in the pallet system designed based on XT technology, $N_{p}^{*}$ and $T_{c}^{*}$ respectively are 7 and $8.46\left[\frac{s}{m}\right]$. These values give the minimal drive unit energy consumption about 6.894 kilo joules in every 160 seconds of the production $B 2, B 1, C, A$. This minimal power consumption is significantly lower than $17.547\left[\frac{K J}{c y c l e}\right]$, which is obtained by the design according to the pure lean approach with the minimal number of pallets $N_{p}=5$.

TABLE III

ENERGY CONSUMPTION FOR A GIVE CYCLE TIME BASED ON (13)

\begin{tabular}{cccc}
\hline \hline \multirow{3}{*}{ Tech } & $N_{p}$ & $T_{c}^{\max }\left(N_{p}\right)\left[\frac{s}{m}\right]$ & $E\left(N_{p}, T_{c}^{\text {max }}\right)\left[\frac{K J}{c y c l e}\right]$ \\
\hline & 6 & 1.625 & 3.161 \\
X85 & $\mathbf{7}$ & $\mathbf{4 . 7 5}$ & $\mathbf{0 . 8 7 1}$ \\
& 8 & 5.75 & 0.989 \\
& 9 & 7.00 & 0.991 \\
& 10 & 8.5 & 0.927 \\
& 11 & 10 & 0.883 \\
\hline & 6 & 2.94 & 6.006 \\
& 7 & 5.3 & 4.083 \\
XK & 8 & 7.65 & 3.352 \\
& 9 & 11.76 & 2.140 \\
& 10 & 14.7 & 1.882 \\
& 11 & 17.06 & 1.855 \\
& $\mathbf{1 2}$ & $\mathbf{1 9 . 4 1}$ & $\mathbf{1 . 8 3 7}$ \\
& 13 & 20 & 2.207 \\
\hline & 5 & 2.3 & 17.547 \\
XT & 6 & 5.00 & 10.312 \\
& $\mathbf{7}$ & $\mathbf{8 . 4 6}$ & $\mathbf{6 . 8 9 4}$ \\
& 8 & 10 & 7.631
\end{tabular}

\section{CONCLUSION}

In this paper two important design questions for pallet systems were investigated. The first question conjectured the equality of the minimal number of pallets $N_{p}^{m i n}$ with the number of pallets $N_{p}^{*}$ giving the minimal drive unit energy consumption $E^{*}$. The second question demanded the method of green control gaining $E^{*}$ provided that the conjecture was disapproved. Both these questions were answered by devising an optimization model in which the mean value of the energy consumption was the objective function, and the system design specifications were the constraints. The result of this model for three cases of pallet systems not only disapproved the conjecture, but also proposed a promising reduction of the energy consumption for $N_{p}^{*}>N_{p}^{\text {min }}$.

The machine failures, and the time complexity of the models due to the variable permutation matrix are not addressed in this study. An attempt to resolve the aforementioned problems, thus, can result in the further enhancement of the presented models. Furthermore, devising a control policy to reduce energy consumption in processing machines can be considered as another interesting subject for the future works.

\section{REFERENCES}

[1] A.M. Deif, "A system model for green manufacturing", Journal of Advances in Production Engineering \& Management, Vol. 6, No. 1, 2011.

[2] M.P. Groover, "Automation, production systems, and computerintegrated manufacturing", Second Edition, Prentice Hall International 2001

[3] T. Murata, "Petri Nets: Properties, analysis and applications", Proc. of the IEEE, Vol. 77, No.4, pp. 541-580, 1989.

[4] C.V. Ramamoorthy, G.S. Ho, "Performance evaluation of asynchronous concurrent systems using Petri nets", IEEE Transactions on Software Engineering, Vol.6, pp. 440-449, 1980.

[5] W.J. Hopp, and M.L. Spearman, "Factory physics: Foundations of manufacturing management", 2nd edition, IrwinlMcGraw Hill, NY, 2000

[6] J.D. Little, "A proof of the queueing formula $L=\lambda W$ ", Operation Research, Vol. 9, No. 3, pp. 383-387, 1961.

[7] J. Li, D.E. Blumenfeld, N. Huang, and J.M. Alden, "Throughput analysis of production systems: recent advances and future topics", International Journal of Production Research, Vol. 47, No. 14, pp. 38233851, 2009.

[8] Y. Frein, C. Commault, and Y. Dallery, "Modeling and analysis of closed-loop production lines with unreliable machines and finite buffers", IIE Transactions, Vol 28, pp. 545-554, 1996.

[9] S.B Gershwin and L.M. Werner, "An approximate analytical method for evaluating the performance of closed-loop flow systems with unreliable machines and finite buffers", International Journal of Production Research, Vol. 45, pp. 30853111, 2007.

[10] M.S Han, D.J Park" Performance analysis and optimization of cyclic production line", IIE Transactions, Vol 34, pp. 411-422, 2002.

[11] S. Biller, S.P. Marin, S.M. Meerkov, and L. Zhang, "Closed bernoulli production Lines: Analysis, Continuous Improvement, and Leanness", IEEE Transactions on Automation Science and Engineering, Vol. 6, No. 1, pp. 168-180, 2009.

[12] J. Magott, "Performance evaluation of concurrent systems using Petri nets", Information Processing Letters, vol. 18, No. 1, pp. 713, 1984.

[13] T. Yamada, S. Kataoka, "On some LP problems for performance evaluation of timed marked graphs", IEEE Transactions on Automatic Control, Vol.39, pp. 696-698, 1994.

[14] O. Korbaa, H. Camus, and J.C Gentina "A new cyclic scheduling algorithm for flexible manufacturing systems", The International Journal of Flexible Manufacturing Systems, Vol 14, pp. 173-187, 2002.

[15] F. Chauvet, J.W. Herrmann, and J.M. Proth, Optimization of cyclic production systems: a heuristic approach, IEEE Transactions on Robotics and Automation, Vol 19, No. 1, pp. 150-154, 2003.

[16] T. Hsu, O. Korbaa b, R. Dupas, and G. Goncalves, "Cyclic scheduling for F.M.S.: Modelling and evolutionary solving approach", European Journal of Operational Research, Vol. 191, pp. 464-484, 2008.

[17] M. L. Pinedo, " Scheduling: Theory, algorithms, and systems", Third Edition, Springer, ISBN: 978-0-387-78934-7, 2008.

[18] M. Mashaei, B. Lennartson, F. Sannehed, and G. Abbestam, " Optimal design of a decoupled multiple-loop pallet system for flexible cyclic manufacturing plants", CASE, IEEE Conference on Automation Science and Engineering, 2010.

[19] http://www.flexlink.com. 